\title{
Correction to: The Role of Skin Thickness in the Choice of a Rhinoplasty Technique for Thin-Skinned Patients: Analysis of Long-Term Results and Patient Satisfaction
}

\author{
Mauro Barone ${ }^{1,2,3} \mathbb{D} \cdot$ Annalisa Cogliandro ${ }^{1,2} \cdot$ Rosa Salzillo $^{1} \cdot$ Silvia Ciarrocchi $^{1}$ • \\ Vincenzo Panasiti $^{1} \cdot$ Rosa Coppola $^{1}$ - Vito Russo ${ }^{1} \cdot$ Stefania Tenna $^{1}$. \\ Paolo Persichetti ${ }^{1,2}$
}

Published online: 8 June 2020

(C) Springer Science+Business Media, LLC, part of Springer Nature and International Society of Aesthetic Plastic Surgery 2020

\section{Erratum to: Aesthetic Plast Surg https://doi.org/10.1007/s00266-020- 01763-6}

The authors of this article wish to make the following clarification:

The FACE-Q is a trademarked patient reported outcome instrument. The "Satisfaction with Nose" scale described and reproduced in this article is similarly trademarked; the copyright is retained by the Q-Portfolio. If readers would like to use the FACE-Q in research or clinical practice, they are directed to www.qportfolio.org to obtain a license permission from the copyright holders. Reproduction of the FACE-Q in publications without prior permission is not permitted.

Publisher's Note Springer Nature remains neutral with regard to jurisdictional claims in published maps and institutional affiliations.
The original article can be found online at https://doi.org/10.1007/ s00266-020-01763-6.

Mauro Barone

maurosabbarone@gmail.com

1 Plastic and Reconstructive Surgery Unit, Campus BioMedico University of Rome, Via Alvaro Del Portillo 200, Rome, Italy

2 Research Group "To be and to appear: Objective Indication to Plastic Surgery" of Campus Bio-Medico University in Rome, Rome, Italy

3 Institute of Philosophy of Scientific and Technological Activity, Campus Bio-Medico University of Rome, Rome, Italy 